\title{
DIALCAT: Diabetes as an Accelerator of Cognitive Impairment and Alzheimer's Disease, Comprehensive Approach and Adherence to Treatment
}

\author{
Cristian Barrué ${ }^{1}$, Roger Vila ${ }^{1}$, Antonio B. Martinez ${ }^{1}$, Mario Martín ${ }^{1}$, Javier Vázquez-Salceda $^{1}$ \\ Rafael Simó ${ }^{2,4}$, Marta Barroso ${ }^{1}$, Ulises Cortés ${ }^{1,3}$ \\ ${ }^{1}$ Universitat Politècnica de Catalunya, \\ Computer Science Department, Barcelona, \\ Spain \\ ${ }^{2}$ Vall d'Hebron Institut de Recerca (VHIR), Barcelona, \\ Spain \\ ${ }^{3}$ Barcelona Supercomputing Center, Barcelona, \\ Spain \\ ${ }^{4}$ CIBERDEM (Instituto de Salud Carlos III), Barcelona, \\ Spain \\ \{cbarrue,mbarroso\}@cs.upc.edu, rafael.simo@vhir.org
}

\begin{abstract}
In this article, we present the design of an mHealth system to support senior citizens with chronic diseases in managing their adherence to pharmacological treatment. The DIALCAT system consisting of a smart pill dispenser and a mobile application will be tested in a clinical study for 18 months by patients suffering diabetes with mild cognitive impairment. The clinical trial aims to study the effect of diabetes as an accelerator of cognitive impairment and Alzheimer's disease and the impact of adherence to treatment. DIALCAT is a RIS3CAT project co-funded under the European ERDF programme.
\end{abstract}

Keywords. eHealth, active and assisted living, diabetes, mild cognitive impairment, treatment adherence, mHealth.

\section{Introduction}

According to the World Health Organisation [41], 47M people around the world have some form of dementia, for which there is no effective intervention, to halt or reverse the progressive cognitive impairment. As Europe's population is ageing, long-term care for elderly citizens will become an increasing cost for society. To manage these transition healthcare policies in the EU and individual Member States are heavily focused on extending the independent life of the elderly, with the dual aim of increasing their quality of life (QoL) and reducing the costs of care.

At this time exists enough robust epidemiological data [35] showing that patients with diabetes mellitus type 2 (DM2) present between 2-3 more prevalence of severe cognitive impairment and Alzheimer's disease (AD) than the general population compared by age. This circumstance poses a severe social and health problem. This population generates a bill of approximately 5,500 million euros to Spain's healthcare system.

The diabetic patients with mild cognitive impairment $(\mathrm{MCl})$ is a group with a particular predisposition to develop $A D$ and are generally composed of polymedicated patients. Moreover, cognitive impairment may imply for this kind of 
patients, among other things, a lack of treatment adherence and errors in drug management that may have serious consequences.

Treatment adherence is understood, as the measure in which a person's actions - medicine intake, to follow a diet, and/or to implement lifestyle changes - correspond to the health professionals' recommendations. It is undeniable that many patients have difficulties in following the recommendations of their doctor, severely compromising the effectiveness of the treatment and creating a critical problem both from the health and QoL point of view as well as in economic terms.

The DM2 patients are the paradigm of polymedicated patients and if, also, they present a certain degree of cognitive decline, forgetfulness and mistakes in drug management increase significantly [37]. Providing the means to facilitate and increase adherence to the theoretical treatment will have a tremendous social-sanitary impact.

The DIALCAT project is an initiative funded by the European ERDF programme that proposes a comprehensive multidisciplinary approach to the problem that includes observational clinical research and a pilot intervention study to improve metabolic control in DM2 patients with $\mathrm{MCl}$. One of the objectives of the project is to improve treatment adherence of these patients and to detect possible adverse effects that may arise from polymedication, in particular, a decrease in metabolic control, through an interventional clinical trial with the ultimate goal of avoiding or delaying as much as possible the appearance of the AD.

DIALCAT proposes as an innovative element, the development and validation of an electronic drug dispenser that allows for adherence support and monitoring. This device is integrated with a mHealth application developed and validated during the same trial that will facilitate treatment management by patients and caregivers interacting directly with other involved stakeholders (clinicians and pharmacists) in order to increase the concordance and, consequently, efficiency in the improvement of metabolic control. This paper will focus on the description of DIALCAT's mHealth system and the interventional pilot study.

\subsection{Plan of the Work}

The plan for this paper is the following, in Section 2 we will detail the treatment adherence problem, in Section 3 we will address related work explaining relevant eHealth platforms implemented to give support to treatment adherence. Section 4 will develop the DIALCAT architecture, deployment and its goals. In Section 5 we explain the objectives of the pilot study and give insight information about the DIALCAT stakeholders.

Section 6 is devoted to discuss our conclusions and comment the future work.

\section{Adherence}

Chronic diseases are a significant and growing problem in the population. They are by far the leading cause of mortality, representing some $86 \%$ of all deaths in the EU. Adherence to long-term therapy for chronic illnesses in developed countries averages $50 \%$. In developing countries, the rates are even lower. It is undeniable that many patients experience difficulty in following treatment recommendations [40]. Individuals suffering from one (or more) of chronic diseases will receive complex polypharmacy therapy. High pill burden decreases adherence to drug therapy, resulting from the need to take a large number of pills or other forms of medication on a regular basis. Adherence rates are typically higher in patients with acute conditions, as compared to those with chronic conditions, with adherence dropping most dramatically after the first six months of therapy [26] and in prophylaxis.

Poor adherence to long-term therapies severely compromises the effectiveness of treatment making this a critical issue in population health both from the perspective of quality of life and health economics. Treatment non-adherence can be considered a significant health care problem. This fact is especially true for patients with a chronic illness where adherence is a crucial factor in the effectiveness of a therapy [6]. Concordance and adherence management are of high priority, having a significant effect on the cost-effectiveness of therapy. 
This is especially important where there are disorders with high healthcare costs, such as oncological diseases, psychiatric disorders, HIV, geriatrician disorders or dementia. Initiatives attempting to address medicine non-adherence promote patient involvement in treatment decisions, but there is no single intervention strategy or package of strategies that have been shown to be effective across all patients, conditions and settings.

The ability of patients to follow treatment plans optimally is frequently compromised by more than one barrier, usually related to different aspects of the problem. These include the social and economic factors, the healthcare team/system, the characteristics of the disease therapies and patient-related factors [40]. Trosle has defined non-adherence as "an unavoidable by-product of collisions between the clinical world and the other competing worlds of work, play, friendships and family life" [39]. Interventions using applied high-technology show potential for supporting therapy adherence in patients with diseases that require a polypharmacological treatment. Pharmacist care could help to reach optimal cooperation between patients, the healthcare professional using high technology interventions, as one of the main objectives is to improve the rate of patient adherence in long-term therapy. Treatment non-adherence is an expensive and potentially deadly problem, resulting in 89,000 deaths and 100 billion US dollars per year in unnecessary hospital costs, in the USA.

It has been estimated that there are 194,500 deaths a year in the EU due to miss-dose and non-adherence of prescribed medication [27]. Non-adherence is estimated to cost the European Union $125 \mathrm{~B} €$ annually [27]. Unused medications returned in the UK are incinerated. In the UK alone $10 \%$ of all drug waste is incinerated each year, valued at about $369.6 \mathrm{M} €$. Studies consistently find significant cost-savings and increases in the effectiveness of health interventions that are attributable to interventions for improving adherence [36].

Without a system that addresses the determinants of adherence, advances in biomedical technology will fail to realise their potential to reduce the burden of chronic illness. Interventions that target adherence must be tailored to the particular illness-related demands experienced by the patient, mainly because most of the care needed for chronic conditions is based on patient self-management (usually requiring complex multitherapies) and changes in the lifestyle.

\section{Related Work}

Despite technical advances in Telehealth, Sensors, Cognitive Systems and Social Networks, systems controlling medical non-adherence are still not integrated and embedded in the care process, especially for medication adherence fulfilment and assessment at home.

\subsection{Methods of Assessing Adherence}

Accurate assessment of adherence behaviour is necessary for effective and efficient treatment planning, and for ensuring that changes in health outcomes can be attributed to the recommended regimen. Indisputably, there is no gold standard for measuring adherence behaviour [9]. Traditional adherence assessment methods that one can find in standard clinical practice often yield inaccuracies when used as standalone methods [16]. There are three main approaches: the patient or caregiver self-report, review of refill records and pill counts. Pharmacist assessment using questions would be included in the self-report method.

1. Self-report: clinicians traditionally rely upon self-report to assess medication adherence to treatment. During the visit, the patient/caregiver is asked a set of direct questions regarding medication use and treatment concordance (e.g. diet, physical activity, etc.). Most of times users answer positively even if it is false, to avoid alienating their provider and because of discomfort in sharing difficulties associated with medication use. This interview method has proven to be the less reliable [10]. An alternative interviewing method to obtain more information [33] consists in formulating open-ended questions like Will you tell me how you take your medications?. An example of 
354 Cristian Barrué, Roger Vila, Antonio B. Martinez, Mario Martín, Javier Vázquez Salceda, Rafael Simó, et al.

a self-assessment validated clinical screening tool is the Morisky scale [24].

2. Prescription Refill Methods: Are partially useful for assessing medication adherence if the patient purchases their medications from a single source or an electronic recipe is available allowing a track of the purchase. Information obtained like this is generally inadequate [11] as obtaining information about refilling, or prescription renewal does not provide information about real consumption.

3. Pill Counts: Although it was considered a useful method to assess adherence, it has proven to be unreliable, tending to overestimate adherence [31].

\subsection{Types of Non-Adherence}

Treatment non-adherence causes vary among patients and are broadly categorised as unintentional or intentional. Unintentional non-adherence involves intending to take a medication or following a treatment pattern as instructed but failing to do so for some reason (e.g. forgetfulness, carelessness). Unintentional non-adherence is influenced by patient characteristics, treatment factors and patient-provider issues. In contrast, intentional non-adherence involves making a reasoned decision not to follow treatment as instructed based on perceptions, feelings or beliefs. Intentional non-adherence reflects a rational decision-making process by the patient whereby the benefits of treatment are weighed against any adverse effects of the treatment. Broadly characterising non-adherence may oversimplify the complexities involved in non-adherence, but it is practical and illustrates that mitigating non-adherence requires different interventions.

\subsection{Non-ICT Medication Management Assessment Tools}

Several scales and assessment tools have been designed to evaluate the patient's medication management skills. These scales cover different aspects related to adherence and the medication process for instance:
- Ability to read and understand labels properly.

- Ability to open, withdraw the proper amount of medication and close the medication containers.

- Ability to understand the medication regimens and requirements.

- Ability to follow the correct timing and food/liquid co-ingestion indications.

The validation of some of these scales found that having at least one physical dependency in activities of daily living or cognitive impairment was an independent risk factor for poorer performance [16]. A thorough review study on tools for assessing of medication management skills performed over 44 different instruments (26 invalidated, 18 validated) showed that skills assessed, and methods for assessment, were highly variable [8]. Most validated instruments used a standardised, simulated medication regimen, whereas invalidated instruments mostly used patients' own medications with inadequate detail or instructions to enable standardised administration. To conclude, it can be said that there is no reliable standard scales or tools to measure patient adherence or ability to manage their medication that is widely accepted and recommended in clinical best-practise guidelines. Many different measures have been reported in the literature, and the substantial variations in study design and definitions reported suggest a need to increase standardisation in terminology and definitions of the measure of adherence and persistence to allow comparisons across studies [1].

\subsection{ICT Aids to Adherence Assessment}

Several solutions do exist to support medication adherence; while relatively low technology methods (e.g. pill boxes, pill counts) for adherence assessment are most commonly used, newer ICT tools have been developed.

These include automated pill dispensers, refill reminders and intake reminders and some telehealth approaches. 


\section{Pill Dispensers}

Dozens of solutions are available in the market, from simple and low cost to complex and expensive. They can be classified into three categories:

- Passive: passive pill dispensers include the low technology solutions as pillboxes that enact a storage role in the adherence process. There are many solutions in the market, some of them classifying medications in several doses, along the week and the day to ease intake. The storage style can help to remind which pill corresponds to each intake, but their contribution to medical adherence is completely unproven.

- Reminders: In the reminder category we can find the lowest technology profile pill boxes that have an attached alarm clock that alerts the user when to take medication (MEDGlider [17], MED-Q [20], Med-Time [26])

- Smart: In this category, we can find devices that have capacities included in the previous ones, but have some extra element that provides advanced services. For instance, the Phillips Medication Dispensing Service [28] can retire missed doses that have been left in the tray, provides instructions on how to take medicines or is resistant to energy failures. Pillo [30], offers facial recognition, reminders and the possibility to share dispensing activity with family and caregivers via the internet. Other ICT technologies related with adherence support are targeted to pharmacists like Medical Dispenser [18] that supports them in the preparation of personalized medication blisters. In [23] we explored a pill dispenser associated to a Multi-Agent System able to provide alerts and interact with different actors.

The different solutions presented are among the many technological aids designed to help patients adhere to medication schedules, currently being marketed directly to patients and their caregivers. Designed to be patient friendly and low cost, these technological aids are not supported by adequate reliability and validity data [16]. Controlled trials comparing these systems with conventional methods are lacking.

A patent search was performed using the European Patent Office engine using keywords like treatment adherence or pill dispenser and the authors got more than 800 hits. The patents can be grouped as pill caps for medicine bottles (some with alarms or counters), industrial machines for the pharmacy industry, protection casings for pill blisters, manual devices for blister pill extraction, pill count displays and mechanical pill dispensers.

\section{Mobile apps and Social Networks}

In the market, there are many software solutions oriented to mobile phones. Applications that provide program reminders and dosage configurations for the medications (Pillboxie [29]), reminders for appointments with the doctor and incorporate contact phones (Med O'clock [19]) or include more complex services regarding health management (MyQuest for Patients [25]). The study presented by Dayer [5] about smartphone medication adherence apps examined 160 adherence apps and showed that few apps go beyond the classic reminders providing enhanced services like complex treatment instructions, online databases or provider data entry.

Medical Adherence tools are also reaching the social networks, for instance, the Crohnology social network gathers together stakeholders that can contribute to the treatment of the Crohn disease. CAREGIVERSPRO-MMD [7] is a social network that supports people living with dementia and their caregivers, also provides some treatment adherence support features. Other works are focusing on evaluating the effect of medication reminders to young individuals through the available social networks [21]. OSTEOLINK [15] is an AAL funded project that explored the use of social networks to connect patients and care providers in the osteoporosis context.

Practical social support has been consistently associated with greater medication adherence [34], but studies that associate greater adherence to the use of social networks are needed. 


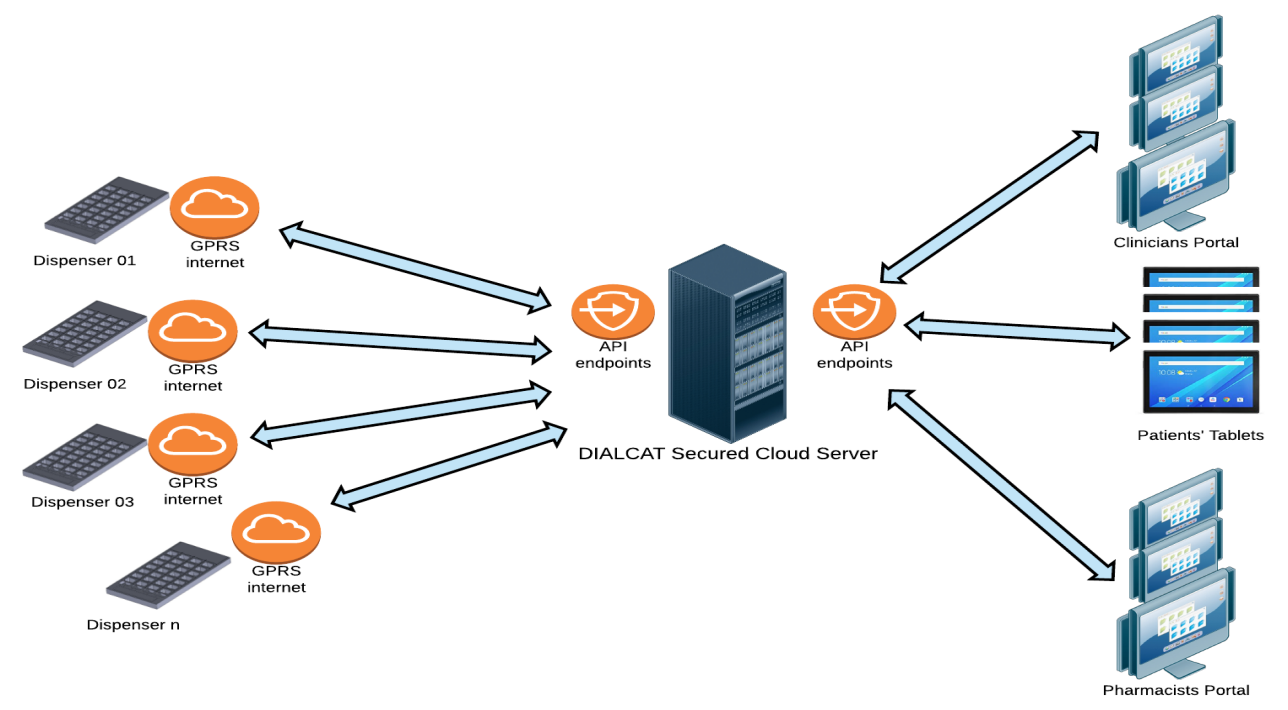

Fig. 1. DIALCAT Architecture

\section{EU Funded Projects}

Many EU funded projects use telehealth platforms to monitor specific pathologies, and some offer medication adherence check through the use of self-reports (CHRONIUS [3], COMOESTAS [4], HEARTCYCLE [12], MONARCA [22]).

Other projects were more focused on improving adherence to treatments, using ICT like the HIVIND [13] project tried to improve adherence to HIV treatment through the use of mobile phone messages or not using ICT and focusing on developing new best-practices or medical trials (QUATRO [32], IMPROVINGADHERENCE [14], $A B C$ [2]).

Only the MEDICATE [38] project included the use of an ICT based pill dispenser to improve adherence from all the studied, but the resulting product never reached the actual standards neither reached the market.

\section{DIALCAT mHealth Platform}

The DIALCAT Project proposes the development of an innovative mHealth platform and well-tailored personalised services to substantially improve chronic patients' medication and treatment adherence (see the architecture in Figure 1). The DIALCAT platform incorporates two elements: i) A Smart pill dispenser that has been developed to provide the medication at the prescribed times, control missed doses with the included sensors, control the drug stock and provide the characteristics of an loT device; ii) DIALCAT mobile app empowering users with the ability to self manage their treatment obtaining tailored information and feedback depending on their treatment concordance.

DIALCAT offers the users the opportunity to self-manage their personalised therapeutic programme under medical supervision at home using the DIALCAT platform. This can lead patients to achieve their treatment goal(s) in their preferred environment, improving motivation, and providing an objective assessment of the personalised protocol and its outcomes during all the process. Furthermore, it may provide a unique opportunity to gather a large amount of data on adherence to therapeutic regimen outcomes for extended periods of time, that can be shared 
to increase knowledge on users' lifestyle and evolution, to predict potential risks or benefits and to avoid the cold start effect when new users start to use the DIALCAT platform. The DIALCAT platform will be able to provide timely and precise indicators of patient adherence and produce relevant reports that will help clinicians to improve the personalisation of the treatment and react when necessary.

\subsection{DIALCAT Smart Pill Dispenser}

DIALCAT's smart pill dispenser has a set of capabilities that make it different from what is available in the market beyond the fact that is connected to the DIALCAT system feeding the users' adherence data. The dispenser allows management of the following:

- that drugs that need to be provided at a specific time are in place.

- that drugs to be provided belong to the user linked to the dispenser.

- the information on when and how the pills are removed from a specific cell is recorded.

The device is designed to work in home environments and needs to be refilled weekly. Drug refill service can be performed by trained pharmacists, as they currently do in common practice in the Spanish health system for instance, but can also be performed by trained users. The technological barrier to use the device is very low to make it usable by all kind of users. The design of the device has always considered the use of low-cost materials to make it affordable to as many people as possible when it reaches the commercial phase. The main capabilities of the device are:

- Reminders: the device warns the user about the time of pill in-take along the day. First it does it with blinking lights and later on also uses acoustic alarms when the end of the in-take time slot is getting closer. Connection with the mHealth platform provides updated information about the drug in-take schedule; reminders are dynamically generated depending on treatment changes.
$-7 \times 4$ System: the device has 28 pill cells, seven rows per weekday and four slots per day for the standard in-take doses (breakfast, lunch, dinner, night). The device is uniquely identified for each user and linked to the DIALCAT mHealth platform to get information about the treatment. Drugs are stored following the European standards of medication containment and isolation.

- Adherence control: the installed sensors based on IR LED technology to allow to detect pills with a guaranteed precision of $5 \mathrm{~mm}$. Each cell is controlled by a set of sensors that control the presence of pills (see Figure 2). These sensors allow the DIALCAT dispenser to detect how many times along the day the user takes pills from the device and when does that happen.

- Stock Control: the sensorized box allows to control at every moment which is the status of the device, controlling which cells have been emptied and which have not. This can allow detecting wrong in-takes (empty cells that should not be at a certain moment) or the need to refill the device soon, allowing to alert the user properly or the appointed service provider.

- Soundness: the device provides means of continuously offering its services, even when no electric power is available (thanks to the included battery) or when the internet connection is failing. In any case, the device always allows the user to retrieve the medication. Information is stored, and it is sent when the conditions allow it to keep the mHealth platform updated.

In Figure 3 we can see the firmware flux diagram for the normal operation of the dispenser.

After the design, prototyping and internal validation phases, the device is actually in the production phase.

For the project pilot study, 140 units are being assembled at the time of writing this paper. 
358 Cristian Barrué, Roger Vila, Antonio B. Martinez, Mario Martín, Javier Vázquez Salceda, Rafael Simó, et al.

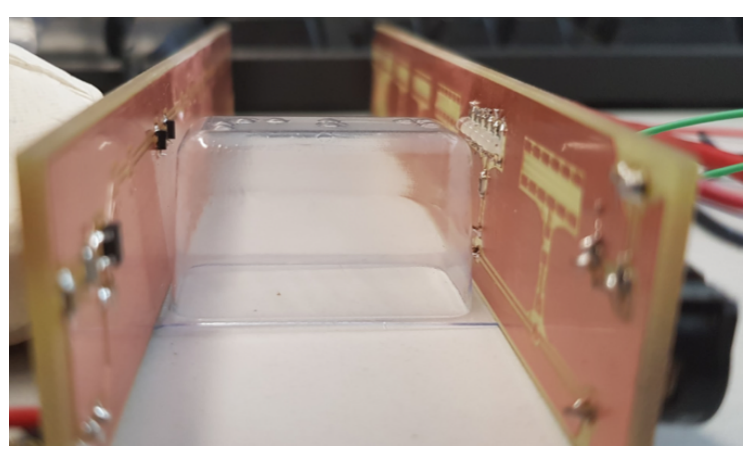

Fig. 2. IR Sensors screening a cell

\subsection{DIALCAT Mobile app}

The mHealth platform allows the integration of information coming from external devices such as the smart dispenser or other (e.g. a glucometer) with the information generated by the platform itself when interacting with the patient and connecting it with the different actors of the therapeutic process: patients, doctors, caregivers and pharmacists. In order to establish this communication, in addition to the digital platform that integrates the data collected automatically, a control panel for doctors and caregivers has been developed, enabling the interaction and the information exchange with regards the therapy. This application allows doctors to be in contact with their patients and at the same time be in contact with other doctors of these patients, allowing a flexible flow of information between clinics in real time that is not available today.

Authorised caregivers/relatives connected to the platform will have information to monitor the patient's health, adherence to the treatment and the evolution of the therapy. At the end of the cycle, we find the pharmacists who will be connected to patients and doctors to receive orders to replenish medication from the dispenser and will validate the treatment schedule for each specific patient. From the control panel, they will be able to check all the medications that should be loaded in the dispenser for each patient as well as those not contained in the dispenser (e.g. suppositories, injections, syrups, etc.). Even if not contained in the dispenser, the device will remind the user to take them. In addition to the platform for the doctors, there is a mobile application that allows patients and caregivers to give feedback on their follow-up of the therapy by answering questionnaires on pharmacological treatment, their lifestyle, diet, exercise, etc. This information is transparently integrated into the platform by generating statistics and reports available to clinicians. The platform also allows to provide reminders about treatment and motivating messages to continue adhering to treatment. The monitoring also reports unexpected effects (mainly due to the drug-drug interaction in the polymedication context), where the system analyses possible action mechanisms that explain this effect. Special adverse effects related to a decrease in metabolic control are of particular importance. The main features of the DIALCAT digital platform are:

- A communication channel between all the therapeutic process stakeholders in the format of a chat system

- A control panel to manage each patient's medication

- A reporting system for adherence and health variables (i.e. glycemia)

- An adherence support system using reminders and personalised messages

- A questionnaire system to collect health data from the patients (i.e. glycemia measures)

- An integration service of external devices like the smart dispenser or glucometers through the use of APIs

- An easy and intuitive user experience

\section{The DIALCAT Study}

The overall objective is to evaluate whether the improvement of metabolic control and hypertension by promoting adherence to treatment and communication between patients and medical equipment can prevent or delay the process of cognitive decline and the conversion to $A D$ in a diabetic patient with $\mathrm{MCl}$. The intervention to be 


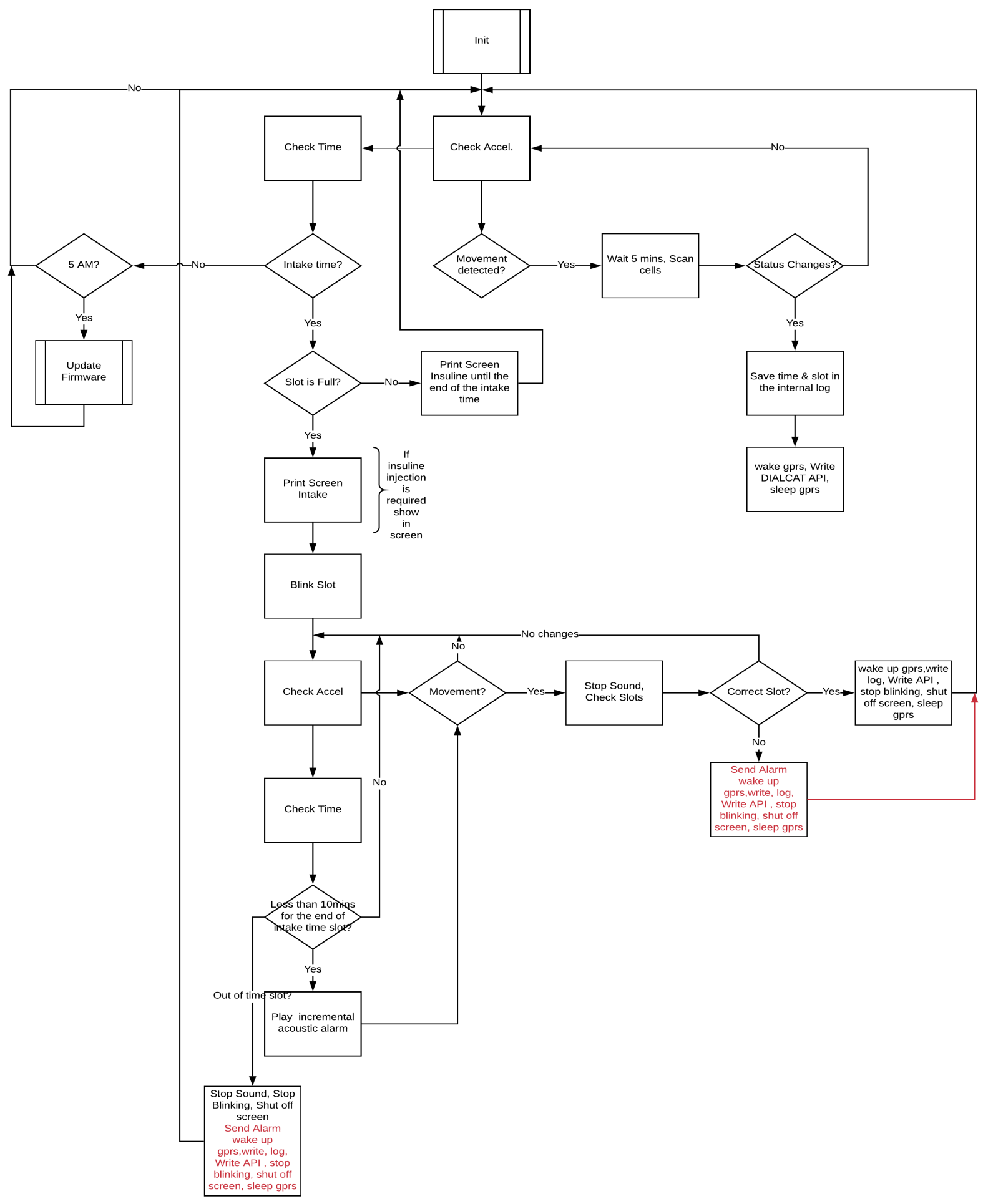

Fig. 3. DIALCAT main algorithm 
applied in the study is the DIALCAT digital platform composed of the smart pill dispenser and the mHealth platform. The study will begin at the beginning of 2019 .

Study Design: Controlled clinical trial, prospective, randomised with parallel groups. The study has been registered at clinical trial.gov.registry.

Primary Objective: To evaluate the effect of the intervention on the progression of cognitive impairment ${ }^{1}$ in an elderly population (60-79 years) with $\mathrm{DM} 2$ and $\mathrm{MCl}$.

\section{Secondary Objectives:}

1. Evaluate if the interventions mentioned above improve metabolic control.

2. Evaluate the effects of these interventions on the conversion rate of $\mathrm{MCl}$ to $\mathrm{AD}$.

3. Compare the effectiveness of the interventions by reducing the memory drop and the conversion rate to $A D$.

4. Identify the clinical and analytical predictors (biomarkers) of conversion from $\mathrm{MCl}$ to $\mathrm{AD}$.

Participants: Patients with $\mathrm{DM} 2$ and $\mathrm{MCl}(\mathrm{n}=$ 174) that will be followed 18 months. Participants will be included in the hospitable centers that participate in the project (Vall d'Hebron Institut de Recerca, Parc Sanitari Pere Virgili, Fundació Althaia, Fundació Univesitaria del Bages, Consorci Sanitari de Terrassa, Fundació Privada Hospital Asil de Granollers, Consorci Hospitalari de Vic, Fundació Docencia i Recerca Mutua de Terrassa).

General Clinical Variables Evaluation: The general clinical variables analyzed will be the following: age, gender, ethnicity, education, anthropometric measurements (body mass index and waist circumference), presence of cardiovascular risk factors (hypertension, dyslipidemia, smoking), alcohol consumption,

\footnotetext{
${ }^{1}$ Assessed by the memory tests included in the cognitive battery
}

pharmacological treatments and family history of dementia. Related with DM2, the following variables will also be recorded: type and time of evolution of diabetes, metabolic control data (basal glycaemia and $\mathrm{HbA1c}$ ) and the presence or absence of complications of diabetes: retinopathy, nephropathy, neuropathy and cardiovascular disease (ischemic heart disease, stroke or peripheral arteriopathy).

Inclusion Criteria: 1) Duration of diabetes of at least 5 years. 2) Age between 60 and 75 years. 3) $\mathrm{MCl}$ (defined criteria).

Exclusion Criteria: 1) AD antecedents. 2) Patients with any type of dementia. 3) History of neurological or psychiatric conditions that can substantially affect cognition, produce sensory impairments or mobility limitations that may prevent or restrict the application or evaluation of the intervention, as well as other important health problems (for example, a recent cardiovascular event clinically relevant, renal disease, oncological treatment).

Randomization: Patients with eligible DM2 will be randomised in a 1: 1: 1 ratio in one of the three branches. The allocation of the treatment will be based on computer randomly generated lists linked to a single number of treatments. For randomisation, a sex-by-sex-swapped sequence and the ApoE genotype will be used. Given that several factors are expected to have synergistic effects, the effectiveness of the cumulative interventions will be studied in 3 parallel arms in which the following interventions will be applied:

Group A: DM2 patients $(\mathrm{n}=58)$ with $\mathrm{MCl}$ who will receive treatment recommended by their primary / endocrinologist.

Group B: DM2 patients $(\mathrm{n}=58)$ with $\mathrm{MCl}$ that apart from the standard treatment will enjoy a DIALCAT smart dispenser that allows the support and monitoring of treatment adherence. 
Group C: DM2 patients $(\mathrm{n}=58)$ with $\mathrm{MCl}$ who, in addition to the dispenser, will receive periodic feedback on their metabolic control and how to improve it by a medical device via the DIALCAT mHealth digital platform.

The ability and possibility of using the necessary technological support (tablet and platform) will not be the criterion of exclusion or assignment to one or the other group. In any case, this will be taken into account at the level of statistical analysis, which will mainly be done by intention to treat. Given that it is expected that the different factors have an additive or synergistic effect, the hypothesis is that the cognitive impairment and the progression of $\mathrm{MCl}$ to $\mathrm{AD}$ would be: Group $1>$ Group 2 > Group 3.

Informed consent and security monitoring: Informed consent will be obtained for each subject, and the centre's ethics committees will evaluate the study for approval.

\section{Conclusions and Future Work}

Seniors with cognitive impairment feel there is a great need for more public awareness of the disease and more support for caregivers. While treatments to reverse or halt disease progression are not available for most of the dementias, and for the foreseeable future, treatment and medication of dementia will remain centred entirely on disease management. Slowing down the rate of cognitive decline and early diagnosis is essential in this as this gives patients the best chance to maintain their cognitive ability.

DIALCAT offers the potential to solve the patient's non-adherence by encouraging self-management of the treatment and making possible the continuity of therapeutic regimen, reducing costs to the patient, the caregivers and the health system.

To improve treatment adherence and to develop target interventions through the mHealth platform channels, it is crucial to address the specific reasons why a patient is not able or willing to execute the treatment plan. From this perspective, intervention messages should be personalised or tailored to address individual needs and beliefs.

The definition of tailoring describes the features that make tailored health messages different from other approaches: It is assessment-based, and as a result, the message can be individual-focused. In other words, tailoring is based on gathering and assessing personal data related to health outcomes or several determinants in order to determine the most effective strategy to meet that personâ ${ }^{\mathrm{TM}}$ s needs.

With these characteristics, a tailored message can provide personal feedback, commands greater attention, is processed more deeply, and is perceived as more likeable by patients than a general message. Because of these possibilities, tailored health messages are also more likely than generic information to be read, remembered, and viewed as personally relevant. Technologies like the smart pill dispenser have the potential to be most useful if the data that generates is processed by all the actors of the health ecosystem.

Moreover, integrated with other health data collected by the mHealth applications and the standard clinical health records, provides a holistic view of the patient's treatment concordance and a support tool to improve decision making.

The next steps in the DIALCAT project are to finalise the industrial production of 140 smart dispenser units and the start of the clinical trial by January 2019. After some months of data collection, the Consortium will be able to process it and start using machine learning techniques to cluster different types of patients with regards adherence and detect connections between adherence rates and disease evolution. The clinical validation of an ICT adherence support system proposed by DIALCAT is a leap ahead in the design of these kinds of technologies.

Moreover, the Consortium will be able to compare adherence rates detected by DIALCAT platform with the ones generated by typical clinical adherence scales (e.g. Morisky [24]) included in $D I A L C A T$ 's clinical trial screening process. 


\section{Acknowledgements}

Authors are partially supported by the project DIALCAT: Diabetes as an accelerator of cognitive impairment and Alzheimer's disease, comprehensive approach and adherence to treatment (DIA) co-funded by European Regional Development Fund (ERDF) of the European Union, within the operative ERDF Catalonian framework 2014-2020. The views expressed in this paper are not necessarily those of the consortium CMMD.

Prof. Cortés is a member of Sistema Nacional de Investigadores (SNI-III), (CONACyT, Mexico).

\section{References}

1. Andrade, S. E., Kahler, K. H., Frech, F., \& Chan, K. A. (2006). Methods for evaluation of medication adherence and persistence using automated databases. Pharmacoepidemiology and drug safety, Vol. 15, No. 8, pp. 565-574.

2. Ascertaining Barriers for Compliance: policies for safe, effective and cost-effective use of medicines in Europe (ABC) (2012). https://cordis . europa.eu/project/rcn/88878/ factsheet/en.

3. Chronius (2012). http: //www. chronious .eu/.

4. COMOESTAS (2010). https://cordis.europa. eu/project/rcn/85409/factsheet/en.

5. Dayer, L., Heldenbrand, S., Anderson, P., Gubbins, P. O., \& Martin, B. C. (2013). Smartphone medication adherence apps: potential benefits to patients and providers. Journal of the American Pharmacists Association, Vol. 53, No. 2, pp. 172-181.

6. DiMatteo, M. (2004). Variations in patients' adherence to medical recommendations: a quantitative review of 50 years of research. Medical Care, Vol. 42, No. 3, pp. 200-209.

7. Dunn, R., Zafeiridi, P., Wolverson, E., \& Paulson, K. (2016). Consulting end-users in the design and usability of CAREGIVERSPRO-MMD: An internet based support tool designed for people with dementia and their caregivers. Alzheimer Europa, pp. PO3.29.
8. Elliott, R. A. \& Marriott, J. L. (2010). Review of instruments used in clinical practice to assess patients' ability to manage medications. Journal of Pharmacy Practice and Research, Vol. 40, No. 1, pp. 36-42.

9. Farmer, K. C. (1999). Methods for measuring and monitoring medication regimen adherence in clinical trials and clinical practice. Clinical Therapeutics, Vol. 21, No. 6, pp. 1074-1090.

10. Hansen, R. A., Kim, M. M., Song, L., Tu, W., Wu, J., \& Murray, M. D. (2009). Adherence: comparison of methods to assess medication adherence and classify nonadherence. Annals of Pharmacotherapy, Vol. 43, No. 3, pp. 413-422.

11. Haynes, R. B., McDonald, H. P., Garg, A., \& Montague, P. (2002). Interventions for helping patients to follow prescriptions for medications. Cochrane database of systematic reviews, , No. 2.

12. Heartcycle (2013). http: //www . heartcycle.eu.

13. HIVIND (2014). https://cordis.europa.eu/ project/rcn/90123/factsheet/en.

14. ImprovingAdherence (2015). https://cordis. europa.eu/project/rcn/105706/factsheet/en.

15. International Osteoporosis Foundation (2010). Osteolink. http://www.aal-europe.eu/projects/ tbreakosteolink/.

16. MacLaughlin, E. J., Raehl, C. L., Treadway, A. K., Sterling, T. L., Zoller, D. P., \& Bond, C. A. (2005). Assessing medication adherence in the elderly. Drugs \& aging, Vol. 22, No. 3, pp. 231-255.

17. MedGlider (2018). https://www.epill.com/ medglider.html.

18. Medical Dispenser (2018). http:// medicaldispenser. cinfa.com.

19. MedOClock (2012). http: //www. medoclock. com.

20. MedQ (2018). http: //lifesavingpillbox . com.

21. Milton S. Hershey Medical Center (2017). Use of social media to improve medication adherence in adolescents and young adults with systemic lupus. https://clinicaltrials.gov/ct2/ show/NCT03218033.

22. MONARCA (2013). https://cordis.europa.eu/ project/rcn/93747/factsheet/en.

23. Moreno, J., Cortés, U., Garcia-Gasulla, D., Gómez-Sebastià, I., \& Álvarez-Napagao, S. (2013). Applying COAALAs to SPiDer. Gibert, K., Botti, V. J., \& Bolaño, R. R., editors, Proc. of 
the $16^{\text {th }}$ Int. Conf. of the Catalan Association for Artificial Intelligence, volume 256 of Frontiers in Artificial Intelligence and Applications, IOS Press, pp. 326-335.

24. Morisky, D., Green, L., Levine, \& D.W. (1986). Concurrent and predictive validity of a self-reported measure of medication adherence. Medical Care, Vol. 24, pp. 67-74.

25. MyQuest for Patients (2018). https://myquest. questdiagnostics.com.

26. National Council on Patient Information and Education (2007). Enhancing Prescription Medicine Adherence: A National Plan. National Council on Patient Information and Education.

27. Pharmaceutical Group of the European Union (2008). Targeting adherence. https://www.pgeu. eu/en/policy/5-adherence.html.

28. Phillips (2018). Automated Medication Dispensing Service. https://www.lifeline.philips.com/ pill-dispenser/health-mdp.html.

29. Pillboxie (2012). http://www . pillboxie.com.

30. Pillo (2018). https://www.pillohealth.com/ technology/.

31. Pullar, T., Kumar, S., Tindall, H., \& Feely, M. (1989). Time to stop counting the tablets? Clinical Pharmacology \& Therapeutics, Vol. 46, No. 2, pp. 163-168.

32. Quality of life following adherence therapy for people disabled by schizophrenia and their carers (QUATRO) (2004). https://cordis . europa.eu/project/rcn/60242/factsheet/en.

33. Raehl, C. L., Bond, C., Woods, T., Patry, R. A., \& Sleeper, R. B. (2002). Individualized drug use assessment in the elderly. Pharmacotherapy: The Journal of Human Pharmacology and Drug Therapy, Vol. 22, No. 10, pp. 1239-1248.

34. Scheurer, D., Choudhry, N., Swanton, K. A., Matlin, O., \& Shrank, W. (2012). Association between different types of social support and medication adherence. The American journal of managed care, Vol. 18, No. 12, pp. e461-7.

35. Simó, R., Ciudin, A., Simó-Servat, O., \& Hernández, C. (2017). Cognitive impairment and dementia: a new emerging complication of type 2 diabetes-the diabetologist's perspective. Acta Diabetologica, Vol. 54, No. 5, pp. 417-424.

36. Simon-Tuval, T., Neumann, P. J., \& Greenberg, D. (2016). Cost-effectiveness of adherence-enhancing interventions: a systematic review. Expert Review of Pharmacoeconomics \& Outcomes Research, Vol. 16, No. 1, pp. 67-84. PMID: 26732615.

37. Smith, D., Lovell, J., Weller, C., Kennedy, B., Winbolt, M., Young, C., \& Ibrahim, J. (2017). A systematic review of medication non-adherence in persons with dementia or cognitive impairment. PLOS ONE, Vol. 12, No. 2, pp. 1-19.

38. The control, identification and delivery of prescribed medication (MEDICATE) (2004). https://cordis.europa.eu/project/rcn/57782/ factsheet/en.

39. Trostle, J. (1988). Medical compliance as an ideology. Social Science \& Medicine, Vol. 27, pp. $1299-1308$.

40. World Health Organisation (2003). Adherence to long-term therapies. evidence for action. https: //www. who.int/chp/knowledge/publications/ adherence_report/en/. $\begin{array}{lcrr}\text { 41. World } & \text { Health } & \text { Organisation } \\ \text { (2016). } & \text { Dementia } & \text { fact } & \text { sheet. } \\ \text { http://www.who.int/mediacentre/factsheets/fs362/en/. }\end{array}$

Article received on 20/10/2018; accepted on 10/02/2019. Corresponding author is Cristian Barrué. 